\title{
Criterios de proyecto para la ampliación significativa del ancho de plataforma de puentes existentes. Ejemplos de aplicación a casos reales de puentes de gran luz
}

\section{Design Criteria for the Significant Widening of the Platform of Existing Bridges. Case Studies of Real Long Span Bridges}

\author{
José $\mathrm{M}^{\mathrm{a}}$ de Villar Luengo ${ }^{\mathrm{a}}$, José M. Simón-Talero Muñoz ${ }^{\mathrm{b}}$ \\ ${ }^{a}$ Ingeniero de Caminos, Canales y Puertos. TORROJA INGENIERÍA S.L., Madrid, España. \\ ${ }^{b}$ Dr. Ingeniero de Caminos, Canales y Puertos. TORROJA INGENIERÍA S.L., Madrid, España. / UPM, España
}

Recibido el 3 de diciembre de 2020; aceptado el 9 de marzo de 2021

\section{RESUMEN}

La ampliación significativa de anchura del tablero de un puente o el aumento de su capacidad portante son actuaciones de envergadura que requieren un análisis cuidadoso en el que, por lo general, se utilizan potentes medios de cálculo y modelos evolutivos que replican las diferentes fases de construcción. Sin embargo, antes de encender el ordenador hay que tener claro para qué.

Se diserta en este este artículo sobre los criterios de proyecto que deben guiar la definición de estas actuaciones de ampliación o mejora estructural. Se incluye también la presentación de cuatro ejemplos de puentes de más de $150 \mathrm{~m}$ de luz principal ampliados últimamente en España. Se presentan los citados criterios de proyecto empleados, los esquemas resistentes elegidos y sus correspondientes "caminos de carga" y, por último, algunas particularidades de los modelos de cálculo utilizados.

PALABRAS ClaVE: Ampliación, rehabilitación, refuerzo, pretensado exterior, hormigón ligero, puente, voladizos sucesivos, atirantado.

(C) 2021 Asociación Española de Ingeniería Estructural (ACHE). Publicado por Cinter Divulgación Técnica S.L. Todos los derechos reservados.

\section{ABSTRACT}

A bridge deck widening or its retrofitting to increase the load capacity are activities that require a careful analysis and usually the use of powerful calculations tools and evolutive structural models to properly define the selected sequence of construction.

This paper is presenting the basis of design to be used as guidelines of the design of the widening or strengthening of major bridges. It is also included the presentation of four examples of widenings of bridges having $150 \mathrm{~m}$ main span length, recently constructed in Spain. The said basis of design, the selected structural scheme and related load paths ant some particular properties of the structural models used for the calculations are presented.

KEYWORDS: Widening, rehabilitation, retrofitting, strengthening, external prestressing, lightweight concrete, bridge, balanced cantilever bridge, cable stayed bridges.

(C) 2021 Asociación Española de Ingeniería Estructural (ACHE). Published by Cinter Divulgación Técnica S.L. All rights reserved.

1.

\section{PRINCIPIOS GENERALES}

Buckland [1], [2] enunció algunos principios generales que, a su juicio, debían servir de guía cuando se trata de incrementar

Persona de contacto / Corresponding author

Correo-e / email: jsimontalero@torroja.es (José M. Simón-Talero) la capacidad portante de un puente en servicio o de proceder a una ampliación significativa de su capacidad. Estos criterios básicos de actuación que, en nuestra opinión siguen plenamente vigentes, se pueden resumir en las siguientes recomendaciones (traducción más o menos literal del original):

- Conozca, lo más ajustadamente posible que pueda, el estado tensional actual del puente; para ello, siga el método de 
inspección-análisis en el que se comparan los resultados de una inspección realizada al puente con un modelo numérico que se utiliza para explicar las diferencias encontradas entre ambos modelos o para tenerlas en cuenta posteriormente.

- Obtenga, lo más aproximado que pueda, los valores y efectos del peso propio y de la carga permanente.

- Defina los valores de los coeficientes de mayoración de acciones y de minoración de la resistencia. Para ello utilice métodos probabilísticos, casos precedentes y el sentido común.

- Procure que el peso de lo que añada sea lo más liviano que pueda. Si es posible o necesario, sustituya elementos existentes por otros de peso más ligero.

- Examine la posibilidad de modificar los "esquemas estructurales" (load paths) o, en su defecto, trate de minimizar la cantidad de refuerzos a ejecutar.

- Recuerde que los trabajos son realizados, a menudo, con ocupaciones temporales de reducida duración: la "constructibilidad" es un elemento clave (key point) en estas operaciones.

- Piense y resuelva primero todo lo relativo a las acciones gravitatorias, pero luego considere mejorar - y no empeorar - el comportamiento del puente frente a las acciones de viento y sísmicas.

- Aproveche y mejore las condiciones del puente con vistas a su mantenimiento y conservación.

- Por último, ENCIENDA EL ORDENADOR: el análisis puede ser sofisticado y complejo, pero debe ser siervo del diseño, y no al contrario, que sea el que lo dirija y justifique.

A estos principios generales que, como se ha dicho, siguen plenamente vigentes, convendría añadir, a nuestro juicio, estas consideraciones adicionales:

- No se olvide de las situaciones temporales durante la construcción, en especial de su estabilidad, ni de los medios auxiliares a emplear y sus efectos, que pueden invalidar la solución propuesta.

- Considere con sentido estructural () y con cierta precaución los repartos de esfuerzos o cargas entre elementos existentes y nuevos.

- Tenga en cuenta la historia de cargas y los posibles repartos de carga no elásticos, si es que puedan ser permitidos.

\section{2.}

\section{ESTRATEGIAS DE AMPLIACION}

Cuando se planea ampliar la anchura de una cierta vía que pasa sobre una estructura en uso y se decide no demoler la estructura existente y sustituirla por otra nueva sino aprovechar, de alguna manera, la antigua, se plantean las siguientes posibilidades, que no son excluyentes unas de otras:

I. Reforzar la estructura existente para que sea capaz de soportar los esfuerzos adicionales que genera la ampliación.

II. Añadir nuevos elementos a la estructura existente de forma que "ayuden" a la existente, repartiéndose los esfuerzos que se generan en la nueva situación.

III. Sustituir "de facto" la estructura existente por otra nueva que recoja el peso de la existente y también las nuevas cargas añadidas.
IV. Añadir una nueva estructura "adosada" de alguna manera a la existente que recoja las nuevas cargas y que funcione de forma más o menos independiente de la existente.

La decisión de adoptar uno u otro depende de múltiples factores sin que se pueda decir, a priori, que uno de ellos sea claramente más conveniente que otro. En general, sí se puede decir que, en casi todos los casos, se dan dos circunstancias comunes:

- La estructura existente colabora, en mayor o menor medida, a soportar las cargas de la situación final.

- Al final, resulta necesario reforzar algunas zonas o elementos de la estructura original, aunque se construya otra nueva que soporte la mayor parte de la nueva carga.

En España se han realizado en estos primeros años del siglo XXI cuatro actuaciones de ampliación significativa del ancho del tablero de un puente de gran luz:

- Viaducto de San Pedro.

Tablero continuo construido por voladizos sucesivos de luz tipo de $150 \mathrm{~m}$; ampliación de 12.0 a $23.6 \mathrm{~m}$ de anchura.

- Puente de Los Santos.

Tablero continuo construido por voladizos sucesivos de luz central de 150 m; ampliación de 12.0 a 24.6 m de anchura.

- Puente de Rande.

Puente atirantado de $400 \mathrm{~m}$ de luz central; ampliación de $2 \times 7.1 \mathrm{~m}$ sobre los $23.4 \mathrm{~m}$ originales.

- Puente del Centenario.

Puente atirantado de $256 \mathrm{~m}$ de luz central; ampliación de un carril sobre los $22 \mathrm{~m}$ originales en primera fase y de $2+2$ carriles en segunda fase, cuya ejecución se prevé iniciar en 2021.

Se encuentra a continuación una breve presentación de las cuatro actuaciones citadas, que luego se utilizarán en apartados posteriores de este artículo, para desarrollar algunos aspectos estructurales y las correspondientes bases de proyecto que sirvieron para su análisis.

a) Viaducto de San Pedro de la Ribera ${ }^{1}$ y puente de Los Santos $^{2}$

En la primera década del siglo XXI se decidió ampliar la plataforma de los viaductos de San Pedro de la Ribera y de Los Santos, situados en Asturias y Galicia. Ambos puentes, que fueron construidos en la década final del siglo XX (en 1994 se abrió al tráfico el viaducto de San Pedro), son de hormigón pretensado y fueron construidos con el método de los voladizos sucesivos; su luz principal es de $150 \mathrm{~m}$ y su anchura original de unos $12 \mathrm{~m}$, que debía incrementarse hasta los 23-24 m de anchura. Es de destacar que, además, el viaducto de San Pedro de la Ribera es curvo y de unos $100 \mathrm{~m}$ de altura de pilas (figuras 1 y 2).

La decisión de proyecto fue, en esencia, mantener el tablero actual pero incrementar su capacidad portante mediante la adición de ciertos elementos longitudinales - núcleos mixtos hormigón-acero y pretensado exterior que, colaborando con la estructura existente, permitieran

1 Proyecto de TORROJA INGENIERÍA

2 Proyecto de FHECOR. 


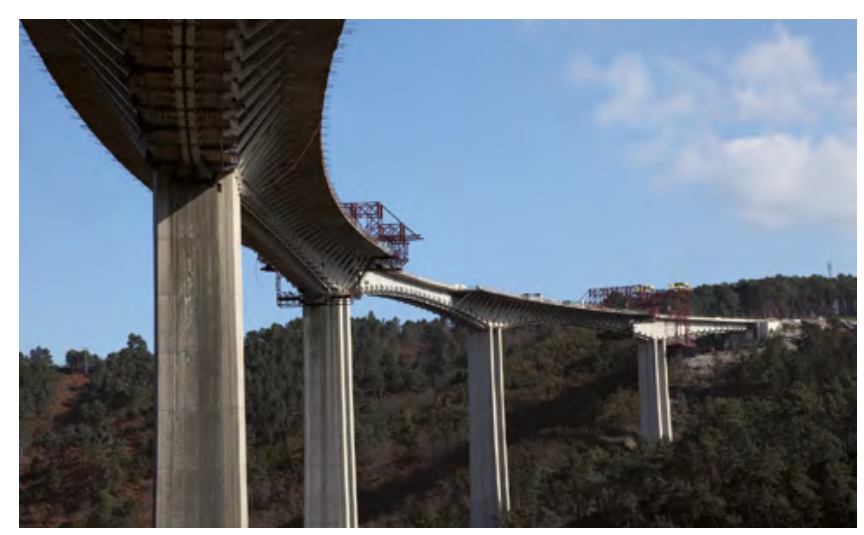

Figura 1. Viaducto de San Pedro de la Ribera.

dicha ampliación [3], [4], [5]. Además, se dotó a la sección transversal de un mecanismo de bielas-tirante que cambió el esquema estructural de la flexión transversal. Es decir, se adoptó la estrategia i+ii mencionadas en la que la estructura existente se complementa con nuevos elementos para conseguir el necesario incremento de su capacidad portante [6].
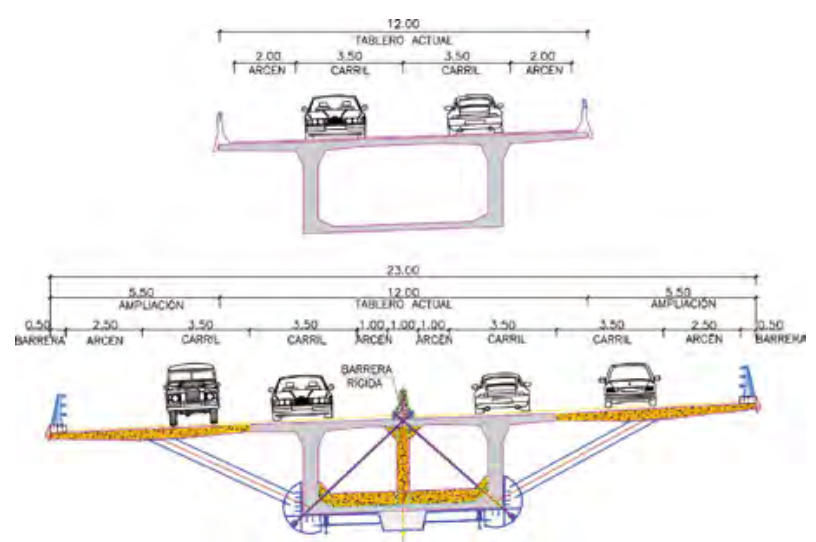

Figura 2. Viaducto de San Pedro de la Ribera. Sección tipo (TORROJA INGENIERIA).

El puente de Los Santos es similar al viaducto de San Pedro de la Ribera. Tiene 3 vamos de $150 \mathrm{~m}$ de luz, $12 \mathrm{~m}$ de anchura de tablero original y fue también construido por voladizos sucesivos, habiéndose aumentado su ancho hasta $24.6 \mathrm{~m}$ con una técnica similar a la empleada en el viaducto de San Pedro (figuras 3 y 4 ).

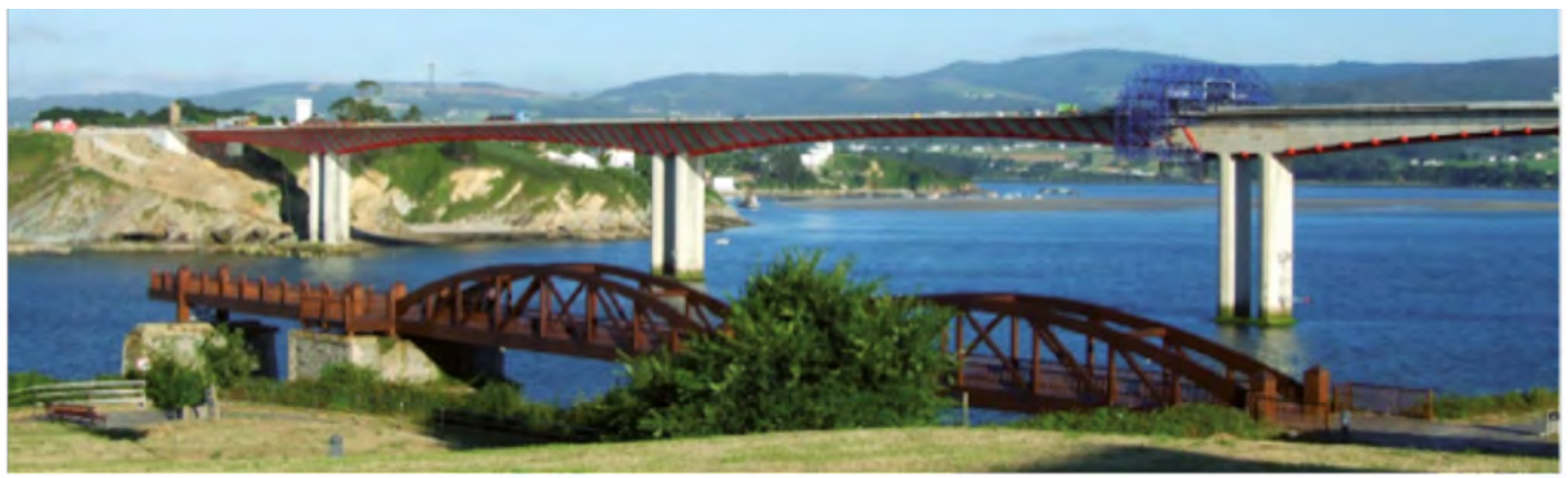

Figura 3. Puente de Los Santos. (FHECOR).

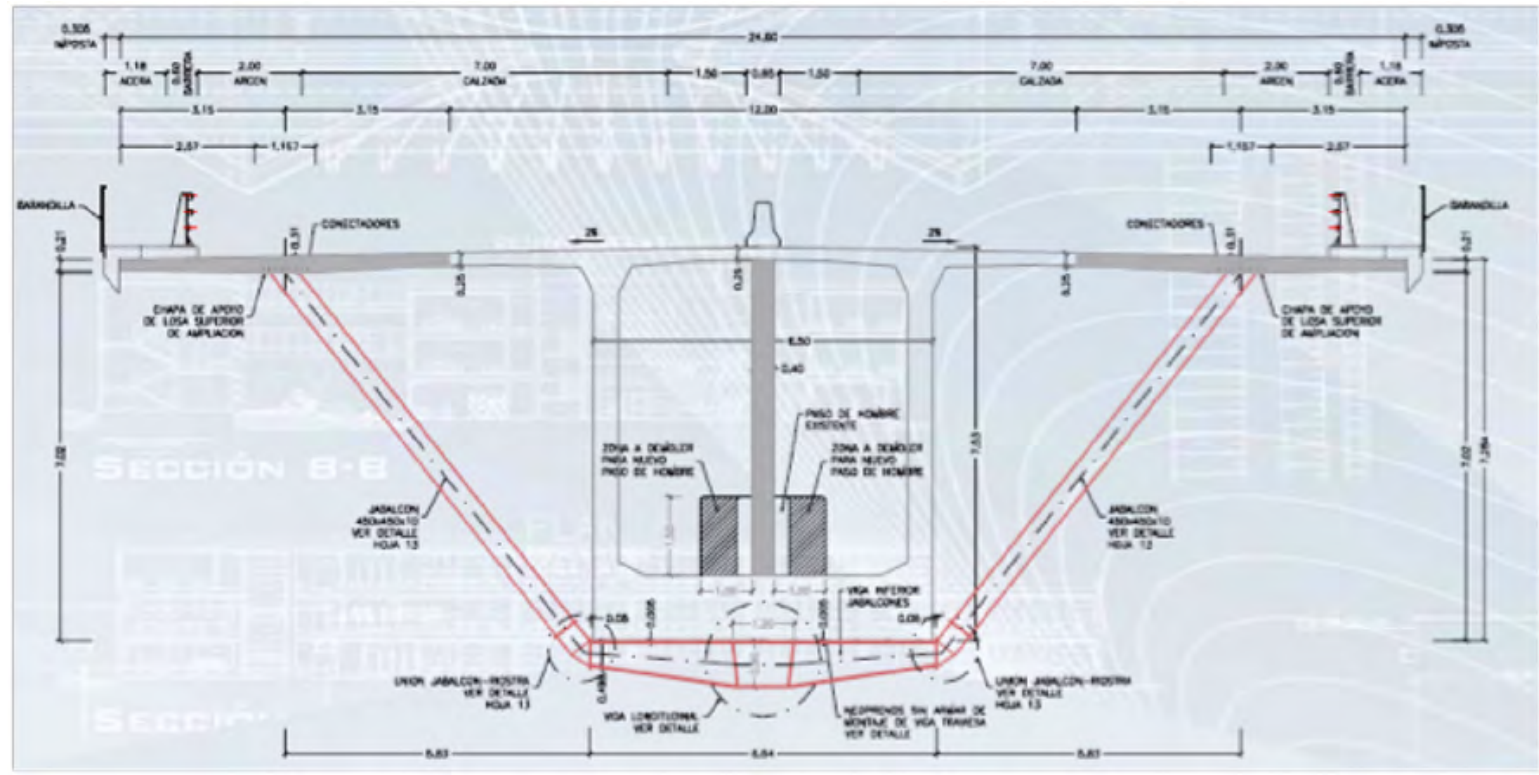

Figura 4. Puente de Los Santos. Sección tipo (FHECOR) 


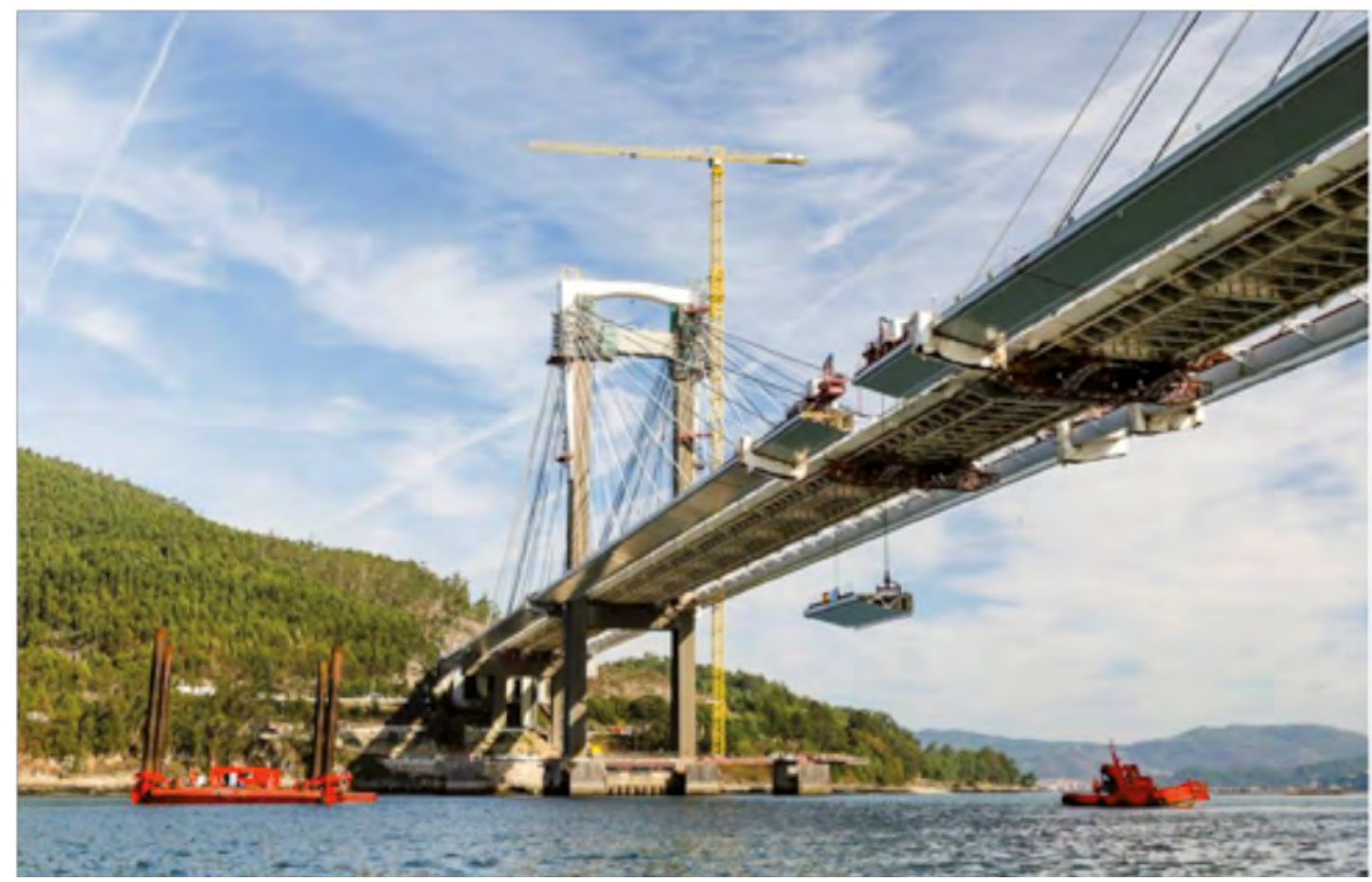

Figura 5. Puente de Rande.

\section{b) Puente de Rande}

En 1977-1978 finalizó la construcción del puente de Rande que con 400 m de luz central era el mayor de España. En 2015 se adjudicó la obra para ampliar la plataforma original de $23.46 \mathrm{~m}$ de anchura con 2 carriles adicionales (figura 5).

Para conseguirlo se decidió suplementar la estructura existente añadiendo sendas nuevas estructuras en los laterales del tablero (figura 6). Estos nuevos "tableros" se soportan con nuevos tirantes que se anclan en la coronación de las torres existentes, que se refuerzan con un elemento metálico en su cabeza. Es decir, se adopta un esquema tipo IV para el tablero (adición de una nueva estructura) y tipo II para las torres (suplemento en las torres existentes) [7], [8] [9].

\section{c) Puente del Centenario 4}

En 2021 se acometerá la sustitución de los tirantes del puente del Centenario en Sevilla. Se trata de un puente atirantado, cuya construcción finalizó en 1991, de 256 m

3 Proyecto de MC-2 y Manuel Juliá.

4 Proyecto de FHECOR+IDEAM, ejecución de la obra en licitación. de luz y $22 \mathrm{~m}$ de ancho de plataforma, cuyo tablero es de hormigón prefabricado (figura 7 ).

Está previsto que, además de cambiar los tirantes, se aproveche para realizar la ampliación de la plataforma desde los 5 carriles existentes a 6, en primera fase (figura 8), y a 10, en una segunda y definitiva fase. La actuación propuesta consiste en retirar los tirantes existentes, previa colocación de unos nuevos que transmiten la carga a unos nuevos fustes que se adosan y trabajan conjuntamente a los pilares que conforman las torres originales del puente. Es decir, el criterio de proyecto es, básicamente, el correspondiente al denominado tipo III+IV (sustitución de la estructura original) puesto que se quitan los tirantes originales y se añaden nuevos, y se dispone una nueva estructura metálica que soporta el tablero de hormigón existente y transfiere su carga a los nuevos tirantes, aunque también se emplea el esquema ii en lo que concierne a las torres en que se comparte resistencia entre los fustes nuevos y los antiguos.

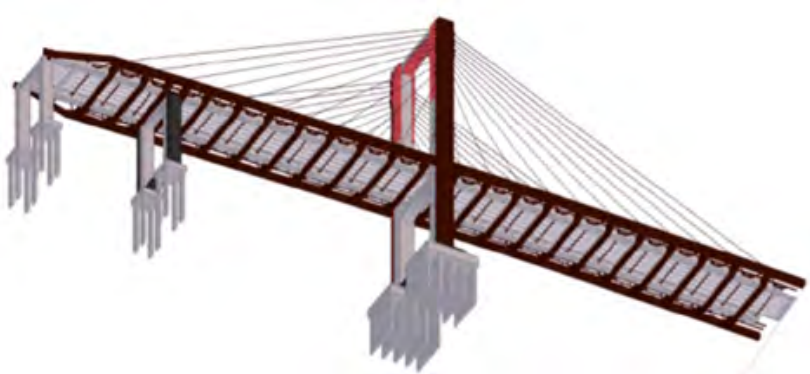

Figura 7. Puente del Centenario. Sustitución de tirantes (FHECOR-IDEAM). 


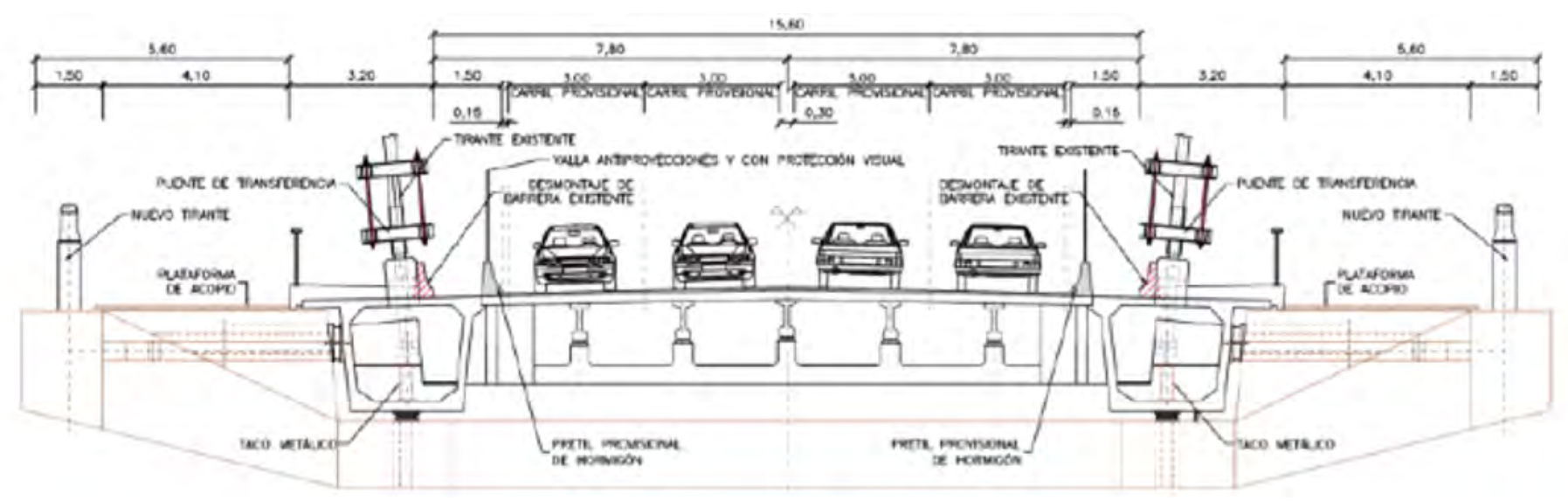

Figura 8. Puente del Centenario. Sustitución de tirantes - Sección tipo $1^{\text {a }}$ fase (FHECOR-IDEAM).

3.

\section{CRITERIOS DE PROYECTO}

A continuación, se pasa revista a algunos criterios de proyecto que, a nuestro juicio, se deben seguir para acometer correctamente (y con "sentido ingenieril") el proyecto de ampliación o de mejora de la capacidad portante de puentes de cierta envergadura. Esta exposición se ayuda con la aplicación práctica que, de cada uno de dichos criterios, se ha hecho en el proyecto de los cuatro puentes ya citados. Entre estas bases de proyecto se destacan las siguientes, que son las que se desarrollan después:

- Conocimiento del estado tensional previo a la actuación de mejora o ampliación.

- Determinación y caracterización de las acciones a considerar.

- Definición de los coeficientes de mayoración de cargas y de minoración de las resistencias a utilizar.

- Elección del modelo de cálculo a emplear. Necesidad generalizada de que sea un modelo evolutivo.

- Criterios para el análisis del reparto de esfuerzos entre elementos existentes y de nueva construcción.

- Definición de las comprobaciones de validación a realizar (ELS y ELU).

- Análisis de las fases constructivas relevantes.

\subsection{Evaluación del estado tensional inicial (Estado 0)}

El conocimiento del estado tensional de la estructura antes de acometer las tareas de ampliación o mejora es imprescindible, como resulta obvio. Sin embargo, el llegar a conocer con cierta exactitud dicho estado es, habitualmente, difícil. Dado que se habla de puentes de envergadura, suelen existir documentos del proyecto y planos as built, por lo que la disponibilidad de documentación que defina la obra original no suele ser un problema.

Sin embargo, el estado tensional de un puente depende en muchos casos de la secuencia constructiva seguida durante su construcción que, muchas veces, es mucho más difícil de conocer con cierta exactitud. Desde luego, se debe desarrollar un modelo estructural que recoja con suficiente nivel de definición la estructura original; así, deberá incorporar no solo la geometría sino también el pretensado, en caso de que exista. También se deberá incorporar al modelo el proceso de ejecución con que se construyó. A título de ejemplo:

- El estado tensional de una cierta sección de un puente construido por voladizos sucesivos no es el mismo que si se hubiera construido por fases sucesivas.

- El estado del tablero, de la torre y de los propios tirantes de un puente atirantado depende del proceso seguido para su construcción y, en mucha medida, de las cargas que se dieran a los tirantes en cada fase de construcción.

Aun así, en muchos casos persiste la incertidumbre en la caracterización del Estado 0 si solo nos basamos en un modelo teórico. Por eso, es muy conveniente realizar alguna toma de datos in situ que nos permita contrastar/validar/ajustar los resultados del modelo al estado real de la estructura. Así, por ejemplo:

- En puentes de hormigón pretensado o metálicos se puede realizar algún ensayo de liberación de tensiones en puntos representativos del tablero que permitan conocer las tensiones existentes en dichos puntos.

- En puentes atirantados se puede (debe) realizar un pesaje de los tirantes que permitirá conocer la carga en ellos. Habrá que ajustar el modelo de cálculo para tener, en todos los tirantes, una carga similar a la carga medida en el pesaje. Hay que destacar que así se consigue saber las cargas en los tirantes, información valiosa para evaluar el estado del tablero que, sin embargo, tiene más incertidumbre en su determinación porque depende del proceso de tesado que se siguió en el momento de su construcción (al que es muy sensible en general, dada la flexibilidad que tienen los tableros de este tipo de puentes) y no solo del estado actual de cargas en los tirantes.

- En puentes muy sensibles a la deformación del hormigón, se pueden extraer testigos para caracterizar su módulo de elasticidad de forma directa, mejor que de forma indirecta basándose en su resistencia.

- Siempre es recomendable realizar una nivelación de la estructura antes de comenzar los trabajos. Si se va a proceder, previamente a la ampliación, a la retirada de algunos elementos del puente (pavimento, barreras, demolición de aceras o de voladizos transversales, ... ) es muy 
conveniente volver a realizar una nivelación del tablero para contrastar las flechas que se produzcan con las esperadas en el cálculo.

Otro aspecto a tener en cuenta es la fluencia del hormigón que modifica y redistribuye a lo largo del tiempo los esfuerzos en puentes hiperestáticos. Así, no se puede olvidar, por ejemplo, que en puentes de voladizos la situación tensional de las secciones de pila a tiempo "infinito" mejora con respecto a las de tiempo "cero" (excepto para lo que corresponde a las pérdidas de pretensado) y ocurre lo contrario en las secciones de centro de vano. Por eso, en puentes de hormigón pretensado, hay que tener en cuenta el factor tiempo a la hora de determinar los esfuerzos y tensiones del Estado 0.

\subsection{Caracterización de las acciones a considerar}

El peso propio y el resto de carga permanente de la estructura existente son componentes intrínsecos del Estado 0 que, por tanto, deben ser conocidos con el mayor grado de precisión que se pueda. En cuanto al peso de los nuevos materiales que se empleen en la ampliación, la máxima es que sean lo más ligeros posibles. En este sentido las ampliaciones con estructura metálica son muy a tener en cuenta, sin que se deba olvidar su mayor deformabilidad, lo que puede resultar en repartos de carga inasumibles. En lo que respecta al hormigón, suele ser conveniente examinar la posibilidad de emplear hormigones fabricados con áridos ligeros (arlita); a este respecto hay que considerar que, aunque el peso específico que se puede alcanzar con ellos es verdad que puede llegar a ser tan bajo como 14 $\mathrm{kN} / \mathrm{m}^{3}$, no conviene considerar menos de $18-20 \mathrm{kN} / \mathrm{m}^{3}$ porque por debajo de este valor la trabajabilidad de estos hormigones es muy dificultosa.

Del pretensado y de las cargas en tirantes ya se ha hablado en el apartado anterior.

Las sobrecargas de uso merecen una consideración específica y diferente según se trate de fases de obra o de puesta en servicio de la obra ya ampliada. Durante la construcción se producen situaciones temporales en que algunos elementos de la estructura pueden estar comprometidos o pueden ser determinantes en el cálculo. Además, durante la construcción se puede tener un cierto control del tráfico que discurre por el puente simultáneamente a las labores de ampliación y, generalmente, con una reducción de carriles relevante. Por estos motivos, se deben definir claramente en el proyecto de ampliación las cargas que se consideren en el cálculo de estas fases. Estas cargas de uso deben ser realistas y compatibles con las condiciones de mantenimiento del tráfico que acepte la autoridad responsable y con las condiciones de ocupación de espacio que se produzcan en cada fase de construcción.

Otra cuestión diferente, pero no baladi, es la situación que se produce en el caso de que las sobrecargas de uso a considerar hayan variado con respecto a las que se empleó en el proyecto inicial de la obra. En tal caso, se debe llevar a cabo la comprobación de los elementos estructurales existentes bajo el efecto de las nuevas cargas de uso, que, generalmente son mayores que las originales lo cual lleva a que sea habitual tener que reforzar la estructura existente. A veces, al realizar los cálculos correspondientes con métodos convencionales se llega a resultados que implican la potencial ejecución de refuerzos que son complicados de ejecutar, muy onerosos, o ambas cosas a la vez. En tales casos se deben emplear métodos de cálculo más sofisticados y herramientas estadísticas que permitan valorar más ajustadamente el valor de las cargas a considerar y los correspondientes coeficientes de seguridad para alcanzar, sin sobrecostes o complicaciones de ejecución, el nivel de fiabilidad correcto. Es de destacar, además, que siempre que se evalúen estructuras existentes se debe considerar correctamente la historia de cargas, de forma que se aplique cada carga sobre la estructura o fase estructural que corresponda; así, por ejemplo, el refuerzo no puede ser considerado como resistente para las cargas de peso propio de la estructura original, si se emplean cálculos elásticos. En muchos de estos puentes importantes la acción del viento resulta muchas veces determinante para la comprobación de los elementos de la subestructura. Por eso, es muy común que se hagan ensayos de túnel de viento para tratar de ajustar el valor de la carga a considerar en el cálculo. En particular, se suelen ajustar los valores de los "coeficientes de arrastre" o "de fuerza" que en las normativas de dimensionamiento suelen estar muy del lado de la seguridad.

Por último, hay que hacer mención a las cargas producidas por los medios auxiliares que se emplean en las diferentes fases de construcción. Es muy habitual que se utilicen carros de avance o de izado, cabestrantes, puentes-grúa cuyo peso ronde los $1000 \mathrm{kN}(100 \mathrm{~T})$ que se sitúan en posiciones muy comprometidas y en fases en que el esquema resistente no es completo y que, por tanto, pueden ser dimensionantes para algunos elementos estructurales.

\subsection{Coeficientes de mayoración de acciones y de minoración de resistencia de los materiales}

Una vez ajustados los valores de las acciones a sus valores característicos o nominales, según corresponda, los coeficientes de mayoración de acciones propios del cálculo en ELU siguiendo el Método de los Estados Límite no tienen, en general, posibilidad de ser modificados ya que los valores adoptados en las Normas se corresponden con el nivel de fiabilidad requerido en ELU y son independientes de la edad de la estructura.

Los coeficientes de minoración de la resistencia de los materiales a emplear en los cálculos en ELU pueden ser diferentes de los que marcan las Normas para obra nueva. Si se realizan ensayos de caracterización de los materiales y de levantamiento de la geometría de los elementos estructurales se puede realizar un análisis probabilístico y adoptar coeficientes diferentes que mantengan el mismo nivel de fiabilidad requerido en las Normas.

Mención especial merece la caracterización del hormigón en dos aspectos. Por una parte, la obtención de la resistencia a compresión de testigos extraídos de la estructura depende mucho del tamaño del testigo y de los áridos, no siendo extraño que resulten valores de la resistencia estimada menores que los esperados al tener en cuenta adecuadamente este "efecto tamaño"; por el contrario, si es muy conveniente conocer la resistencia a tracción estimada del hormigón para acometer los cálculos tensionales en ELS. Por otra parte, no hay que olvidar que el valor de la resistencia a compresión del hormigón no tiene mucha influencia en la estimación de la resistencia a flexión, sino que son preponderantes otros factores como la cuantía y localización de la armadura. 
En lo que se refiere a las estructuras metálicas sí es muy conveniente, en estructuras construidas con acero autopatinable, hacer un estudio de espesores para detectar posibles pérdidas por corrosión y adoptar en el cálculo espesores acordes con la medición efectuada; esto puede llevar a incrementos de la capacidad portante sobre la originalmente estimada (si la previsión de pérdida de espesor es menor que el sobre espesor dado en proyecto) o a reducciones de ella.

\subsection{Modelo de cálculo evolutivo}

El análisis de cualquier ampliación de un puente de cierta envergadura se debe realizar siempre empleando uno, o generalmente varios, modelos de cálculo que son siempre complejos, detallados y que deben reflejar correctamente las diferentes etapas constructivas del proceso. Es importante tener en cuenta las cargas que actúan en cada fase de construcción y sobre qué secciones resistentes lo hacen: por eso, el modelo de cálculo general debe ser "evolutivo".

Además, es muy normal que haya que estudiar fenómenos locales, por ejemplo, en nudos o en conexiones de elementos nuevos a existentes. Es normal, por tanto, emplear también modelos locales para realizar estos estudios. Muchas veces son modelos de elementos finitos que muestran los "caminos de las cargas" (load paths) y que sirven para estimar los repartos de cargas entre elementos o para analizar posibles concentraciones de tensiones de carácter relevante.

En el apartado siguiente de este documento se presentan algunos modelos de cálculo empleados en los proyectos de ampliación que ya se han mencionado.

\subsection{Criterios para el análisis del reparto de esfuerzos entre elementos existentes y de nueva construcción}

Como ya se ha comentado, en casi todas las actuaciones de ampliación hay algunos elementos del puente original y otros de nueva construcción que, a partir de un determinado momento, trabajan solidariamente. Es importante subrayar que este trabajo conjunto se produce solo a partir del momento en que se conectan ambos elementos y, por tanto, todas las cargas precedentes actúan sobre el elemento original; es decir, la colaboración del nuevo elemento es solo "parcial" ya que se produce solo para las cargas "nuevas" actuantes desde ese momento de conexión. Estas nuevas cargas también afectan al elemento original, sobrecargándole y pudiendo hacer que alcancen su límite resistente o funcional. Este es uno de los motivos por los que es muy importante tener en cuenta correctamente la conexión entre ambos elementos, tanto en su topología y dimensiones como en la forma y momento en que entra en carga.

En general, es habitual y recomendable emplear cálculo elástico para determinar el reparto de esfuerzos entre el elemento original y el nuevo. Si se emplean repartos elasto-plásticos o rígido-plásticos con redistribuciones totales o parciales de esfuerzos hay que asegurarse de que las fisuraciones que se producen en los elementos de hormigón bajo las cargas sin mayorar son admisibles, manteniendo la adecuada compatibilidad de deformaciones. Además, estos cálculos no elásticos serán solo válidos en ELU, debiéndose examinar también la "seguridad y durabilidad" en ELS, como se ha comentado. En el apartado siguiente de este documento se presentan algunos ejemplos de reparto de esfuerzos empleados en los proyectos de ampliación que ya se han mencionado.

Según lo expuesto, es muy común que, en una primera aproximación, los resultados del cálculo muestren que algún elemento original alcance una situación inadmisible bajo la acción de las nuevas cargas, aunque parte de ellas sea resistida por la nueva estructura. Por ello es muy común el aplicar una deformación impuesta a la estructura existente antes (o, a veces, después) de conectarla con la nueva que, a modo de, por así decirlo "pretensado", descargue la estructura original antes de aplicarle nuevas cargas. Este es el caso, por ejemplo, de la ampliación de un puente atirantado en que se añaden tirantes nuevos para ayudar a soportar el incremento de cargas; muchas veces se da un tesado a estos tirantes que descarga a los existentes y al tablero y genera margen resistente suficiente para soportar el incremento de esfuerzos posterior.

\subsection{Definición de las comprobaciones de validación a rea- lizar (ELS y ELU)}

Las comprobaciones de validación estructural a realizar en un proyecto de ampliación no pueden ser otras más que las mismas que indican las normas para obra nueva; es decir, se deben regir por el Método de los Estados Límite y deben incluir las comprobaciones en ELS y ELU que sean de aplicación y relevantes en cada caso. Eso sí, hay que tener en cuenta correctamente las particularidades que la coexistencia de elementos portantes antiguos y nuevos supone y que el proceso de construcción evolutivo impone. A continuación, se presentan algunas breves consideraciones sobre algunos de estos aspectos particulares del cálculo.

a) Elementos de hormigón

- En ELS se deben limitar las tensiones y/o fisuraciones durante la construcción, pero teniendo en cuenta también el estado tensional final. Así, si una zona resultara comprimida en situación final se podrán, en general, aceptar tracciones o fisuraciones durante la construcción, puesto que luego se cerrarán dichas fisuras. Esto es válido siempre que, por supuesto, se verifiquen las condiciones de seguridad en ELU en todas las fases constructivas.

- Se puede emplear el método de biela-tirante para comprobar ciertas armaduras. En estos casos habrá que comprobar que la fisuración de esas zonas en ELS es admisible.

- Cuando se amplía la plataforma de un tablero se incrementan bastante las torsiones. El reparto de tensiones tangenciales o de rasantes que producen el cortante y la torsión combinados debe ser examinado con cautela, sobre todo en el caso de que se añada un alma a las ya existentes. Este cálculo se realiza, en general, en ELU y, por tanto, en régimen plástico, pero es aplicable el párrafo anterior y hay que comprobar también la fisuración de las almas en ELS.

- En el caso en que se refuerce un elemento existente con nuevas armaduras hay que comprobar qué deformaciones alcanzan estas nuevas barras para conocer si en ELU trabajan a su límite elástico, o no llegan a él.

b) Elementos metálicos

- En el dimensionamiento de las uniones hay que tener en cuenta correctamente las excentricidades locales que se puedan producir. Esto es común al proyecto de una obra 


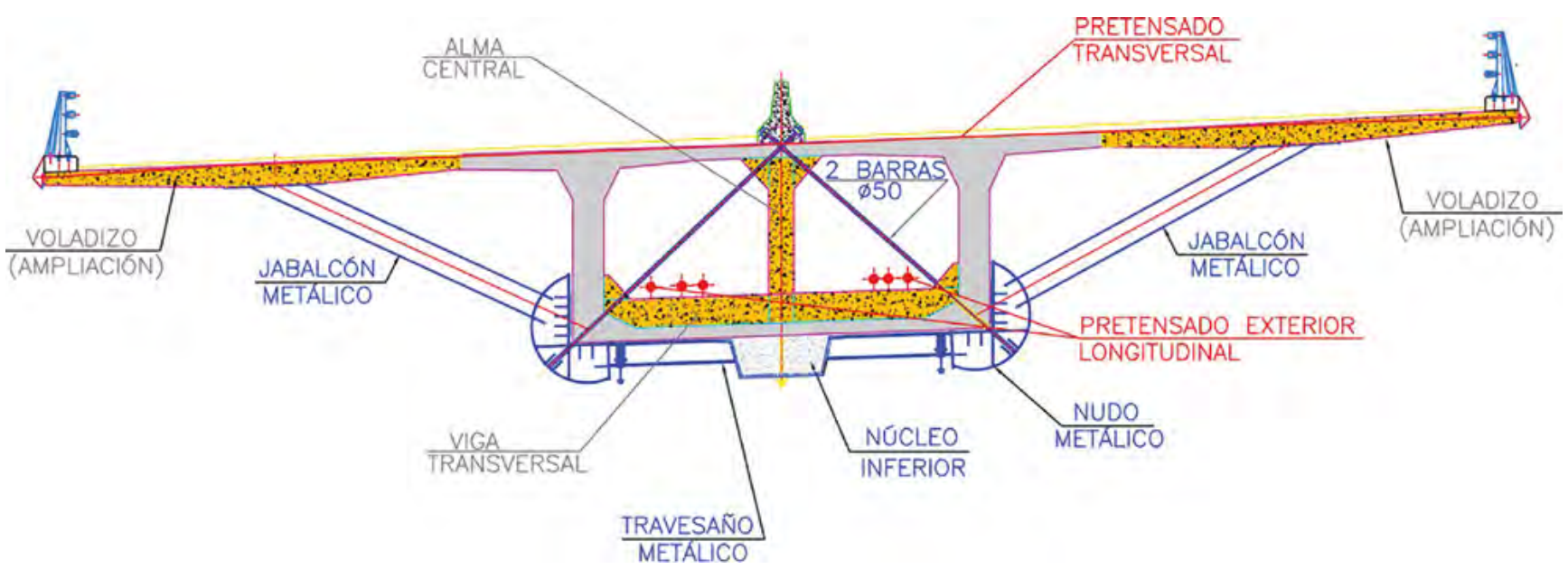

Figura 9. Viaducto de San Pedro de la Ribera. Sección tipo estructural.

nueva, pero requiere un análisis especial en las ampliaciones porque, dado que la geometría de la obra existente es dato, muchas veces no es posible conseguir uniones centradas.

- Se debe limitar la ejecución de soldaduras a elementos portantes principales existentes que estén en carga. Es preferible en estos casos realizar uniones atornilladas. Es de destacar que, en algunos países con tradición en la construcción de acero, este tipo de soldaduras está prohibido o, al menos, se define como poco recomendable y solo aceptable si no es posible realizar una unión atornillada.

c) Tirantes

- Las tensiones máximas en los tirantes se suelen limitar a valores próximos al $45 \%$ de fPU por temas de fatiga. Dado que la ejecución de la ampliación dura un tiempo limitado se puede realizar un estudio ad hoc para fijar cuales la máxima tensión permitida durante la fase de construcción.

- Para poder comprobar los tirantes se debe realizar un pesaje de ellos con identificación clara de a qué fase de construcción pertenece para conocer cuál es el estado inicial (Estado 0) tensional de cada tirante.

\subsection{Análisis de las fases constructivas relevantes}

Ya se ha hablado repetidamente en apartados anteriores de la necesidad de que el proyecto de ampliación contemple adecuadamente el proceso constructivo previsto., Para ello casi siempre es imprescindible emplear un modelo de la estructura evolutivo. Se llama la atención ahora sobre la necesidad de detectar cuales son las fases constructivas más importantes, para estudiarlas con el detalle que merecen. En general, se debe prestar especial atención a:

- Las fases iniciales de la ampliación en que todavía no se suelen haber dispuesto sistemas de refuerzo o nuevos elementos portantes y en los que, además, suelen actuar elementos auxiliares de gran peso.

- Las fases en que se conectan elementos portantes nuevos a otros existentes y se realizan transferencias de cargas de unos a otros.

- Las operaciones de demoliciones parciales de elementos existentes y su posible afección a la capacidad portante local del elemento.

\section{4.}

\section{EJEMPLOS DE APLICACIÓN}

Como ya se ha comentado, se han realizado en España en estos primeros años del siglo XXI $\left({ }^{*}\right)$ cuatro actuaciones de ampliación significativa del ancho del tablero de un puente de gran luz:

$\left({ }^{*}\right)$ Previsto que el proyecto de sustitución de tirantes del puente del Centenario sea ejecutado en 2021-2022)

- Viaducto de San Pedro.

Tablero continuo construido por voladizos sucesivos de luz tipo de $150 \mathrm{~m}$; ampliación de 12.0 a $23.6 \mathrm{~m}$ de anchura.

- Puente de Los Santos.

Tablero continuo construido por voladizos sucesivos de luz central de $150 \mathrm{~m}$; ampliación de 12.0 a $24.6 \mathrm{~m}$ de anchura.

- Puente de Rande.

Puente atirantado de $400 \mathrm{~m}$ de luz central; ampliación de $2 \times 7.1 \mathrm{~m}$ sobre los $23.4 \mathrm{~m}$ originales.

- Puente del Centenario.

Puente atirantado de $256 \mathrm{~m}$ de luz central; ampliación de un carril sobre los $22 \mathrm{~m}$ originales en primera fase y de $2+2$ carriles en segunda fase (previsto que el proyecto de sustitución de tirantes sea ejecutado en 2021-2022).

Se describe a continuación una somera presentación de las cuatro actuaciones citadas en la que se muestra, para cada caso, los criterios de proyecto empleados en cada uno de ellos y como se ha acometido el análisis estructural de algunos aspectos de las ampliaciones proyectadas.

\subsection{Viaducto de San Pedro de la Ribera y puente de Los Santos}

El viaducto de San Pedro de la Ribera es un puente continuo de $750 \mathrm{~m}$ de longitud de canto variable de hormigón preten- 
sado construido con el método de los voladizos sucesivos. Sus 4 luces centrales tienen $150 \mathrm{~m}$ y su anchura original de unos $12 \mathrm{~m}$, que fue ampliada hasta los $23.4 \mathrm{~m}$ de anchura. Es de destacar que, además, el viaducto de San Pedro de la Ribera es curvo y de unos $100 \mathrm{~m}$ de altura de pilas (figura 9).

Se describen a continuación algunos aspectos llamativos del proyecto de ampliación del viaducto de San Pedro de la Ribera ${ }^{5}$. No se incluye ninguna mención adicional al proyecto de ampliación del puente de Los Santos (elaborado por FHECOR) porque, en esencia, se resolvió de una forma bastante similar a la aplicada en el primer puente citado.

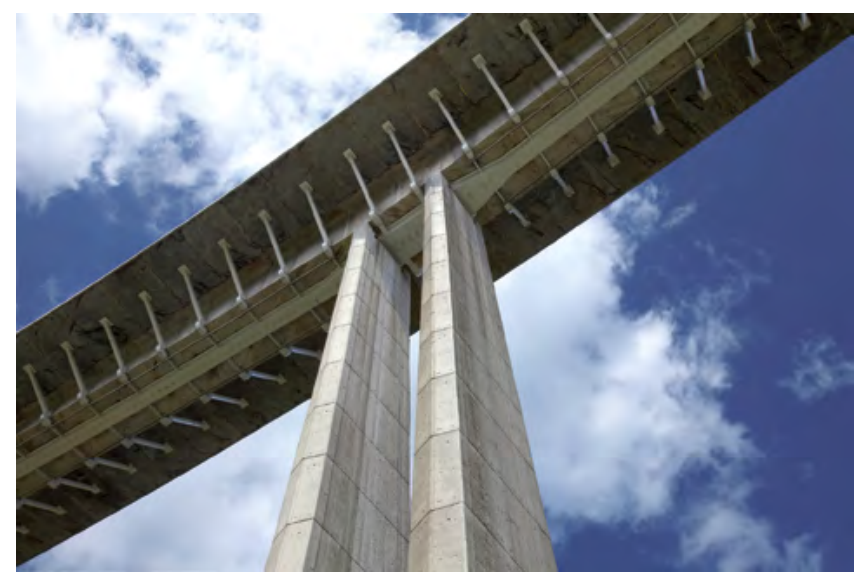

Figura 10. Viaducto de San Pedro de la Ribera. Vista inferior.

La solución adoptada fue reforzar el cajón del tablero existente para que admitiera la totalidad de las cargas debidas a la ampliación, sin recurrir a una estructura exterior. Los elementos básicos que constituían la ampliación y refuerzo del tablero y su función estructural se describen a continuación muy someramente:

- Sistemas de pretensado exterior longitudinal para mejorar la respuesta a flexión del tablero.

- Núcleos mixtos inferiores para aumentar la capacidad portante a flexión, tanto a momentos positivos como negativos.

- Travesaños metálicos que conectan el núcleo con los laterales.

- Alma central que mejora la resistencia a cortante.

- Jabalcones que soportan a compresión los forjados de ampliación.

- Diagonales interiores en el cajón que transmiten la carga de los jabalcones al alma central.

- Pretensado transversal en forjado superior.

- Vigas transversales en forjado inferior que actúan como puntales de compresión.

a) Acciones consideradas. Cargas aplicadas

Debido a los condicionantes tan restrictivos respecto a la subestructura, a la gran altura de pilas y a la ubicación del puente en un valle abierto próximo a la línea de costa, se consideró necesario la realización de ensayos en túnel de viento del tablero y las pilas. Se trataba de estimar con cier-

5 Elaborado por TORROJA INGENIERIA. ta precisión la acción del viento sobre el puente, y compararla con los resultados obtenidos a partir de la formulación propuesta por la IAP. Así, se realizó en el Instituto Universitario de Microgravedad "Ignacio Da Riva", de la Universidad Politécnica de Madrid, la medida mediante ensayos en túnel aerodinámico de las cargas aerodinámicas sobre tablero y pilas del viaducto de San Pedro. Así, se pudo justificar la adopción de unos coeficientes de arrastre un 15$25 \%$ menores que los propugnados en las normativas.

En lo que respecta a los nuevos voladizos transversales se empleó para su construcción hormigón ligero. Después de probar diferentes dosificaciones de árido ligero con vistas a conocer su facilidad de puesta en obra se tomó un peso específico de $22 \mathrm{kN} / \mathrm{m}^{3}$.

b) Modelos de cálculo.

Era requisito de partida del proyecto el que no se tuviera que efectuar ningún refuerzo de las pilas existentes ni de las cimentaciones. Esto fue conseguido por dos motivos: uno, porque la situación dimensionante para las pilas era la de construcción en voladizo, lo cual daba cierto margen para soportar cargas adicionales; dos, porque se han empleado modelos de cálculo sofisticados para su comprobación. En particular, se empleó un modelo 3D de elementos tipo barra en los que se modelizaba también la armadura de los fustes para considerar así los fenómenos de $2^{\circ}$ orden, de no linealidad mecánica y geométrica y de fisuración del hormigón. Además, para el estudio del encepado se empleó un modelo de elementos finitos que incluía la flexibilidad de los pilotes, para poder conocer el ancho eficaz en que era lícito contar como colaborante la armadura situada en las cercanías de cada pilote (figura 10).

Para el cálculo del tablero se empleó un modelo elástico 3D, realizándose un cálculo evolutivo incluyendo las fases de construcción del puente original. Así se tenían en cuenta los efectos de la fluencia y los que se producen en los diferentes esquemas estáticos en que se encuentra el puente en las diferentes fases de construcción

Para el cálculo de la flexión transversal, la distorsión en el cajón, desviadores, traviesas de pilas, esfuerzos en elementos transversales (diagonales, vigas transversales, puntales, jabalcones), así como para el estudio del reparto del cortante entre las tres almas para distintas hipótesis de carga, se utilizó un modelo de elementos finitos, que representaba un vano completo de $150 \mathrm{~m}$ e incluía las condiciones de empotramiento en pilas y de continuidad en vanos adyacentes, desviadores, pretensado transversal, y demás elementos resistentes de la ampliación (figura 11).

c) Reparto de esfuerzos.

Como ejemplo de los criterios de reparto de esfuerzos entre elementos estructurales existentes y nuevos se comenta a continuación el análisis efectuado para conocer la distribución de tensiones tangenciales entre las tres almas de la sección cajón (dos exteriores originales y la nueva central) y el análisis del mecanismo de transmisión de los momentos desde el tablero hacia el fuste de cada pila para confirmar la validez de dicha conexión.

cl) Reparto del cortante y de la torsión entre almas. Mecanismos de flexión transversal.

El refuerzo del cajón existente frente a esfuerzos cortantes se conforma con tres elementos diferentes: por 


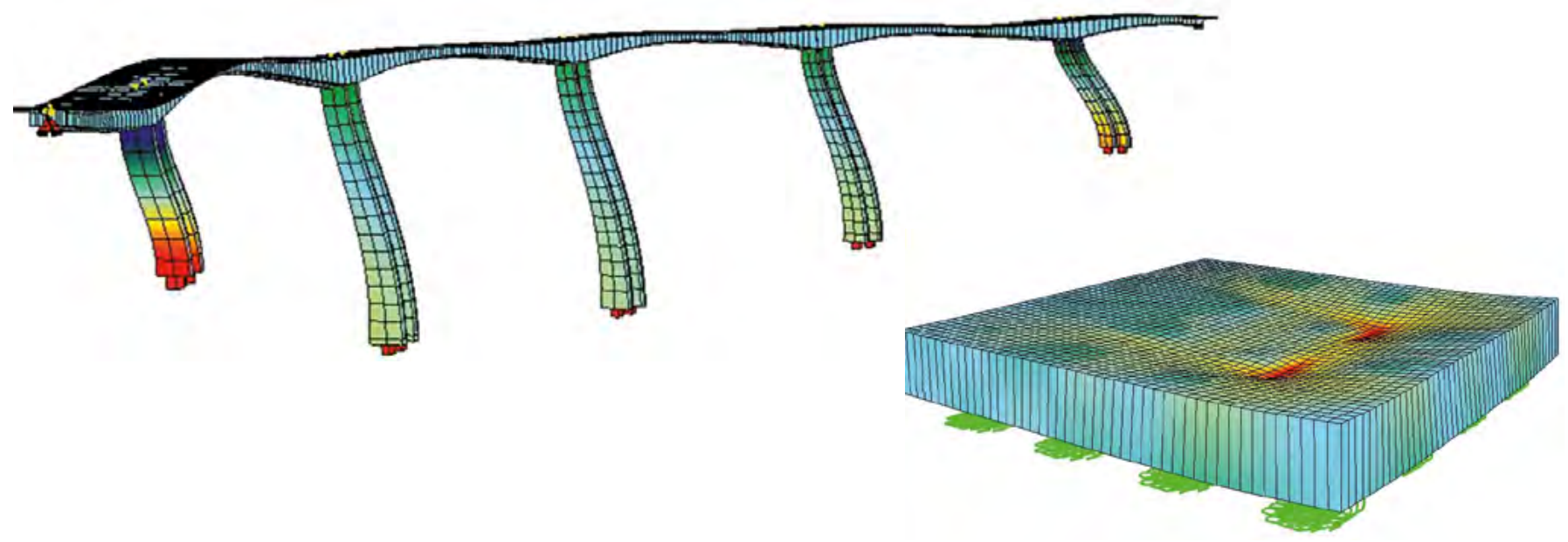

Figura 11. Viaducto de San Pedro de la Ribera. Modelo de la subestructura y de los encepados.

una parte se reducen los cortantes sobre el cajón mediante un pretensado exterior longitudinal que discurre por el interior del cajón disponiéndose cerca de la losa inferior en centros de vano y subiendo a las proximidades de la losa superior cuando se acerca a pilas, presentando unos quiebros que generan cortantes ascendentes sobre el tablero de hormigón; por otra, se añade un alma central, cosida, mediante barras verticales pretensadas, al forjado superior y a la losa inferior del cajón a través del núcleo metálico inferior, y por último, se dispone un sistema de diagonales, formado por series de 4 barras pretensadas de acero de alta resistencia, que se anclan inferiormente en sendos nudos metálicos situados fuera del cajón (adonde llegan los jabalcones metálicos), y superiormente en un tacón dispuesto al efecto en la losa superior del tablero, que posteriormente quedará embebido en la barrera central de hormigón.

El alma central se construye antes de añadir nuevas cargas a la estructura existente y, por tanto, solamente el peso propio del cajón mixto y del alma central se aplica directamente a las almas laterales. El resto de las cargas quedarán aplicadas al conjunto de las tres almas, con un determinado reparto, que se estudió con un modelo de elementos finitos. El objetivo final era el de transferir a esta alma central una parte significativa del esfuerzo cortante total, quedando así aliviadas las almas laterales y permitiendo que estas trabajasen, tanto para ELS como para ELU, en condiciones no peores que las actuales.

Además, el establecimiento del alma central colabora en otros aspectos estructurales: por una parte, alberga las barras pretensadas verticales que anclan el núcleo metálico inferior a la cabeza del cajón a efectos del rasante entre estos dos elementos; por otra parte, el alma central se utiliza para transferir a la parte superior del cajón las cargas transmitidas a su parte inferior por los jabalcones y travesaños que soportan los forjados de ampliación. Para ello se utilizan cuatro barras pretensadas verticales por travesaño, independientes de las barras de cortante antes comentadas, que actúan como barras de cuelgue de las cargas concentradas transmitidas por aquellos travesaños. Las barras diagonales cumplen la misión de, por una parte, al ser tesadas, transferir parte de la carga de las almas laterales, descargándolas, al alma central de nueva ejecución, que está comprimida por las barras verticales tesadas; así se consigue transferir carga de cortante de las almas exteriores al alma central, "liberando" las almas exteriores existentes para que puedan resistir el torsor adicional que genera la ampliación de la plataforma. Por otro lado, estas diagonales recogen la fuerza vertical que producen los jabalcones en los nudos metálicos exteriores, al transmitir las cargas producidas por la nueva carga permanente y la sobrecarga de los nuevos voladizos transversales de ampliación. El esquema resistente transversal se completa con el tirante superior que surge en el forjado superior (figura 12), en el que se dispone un pretensado transversal con vainas planas para compensar las tracciones que se producen en el hormigón.

c2) Conexión del tablero a los fustes de pilas.

Las traviesas de pila existentes en el viaducto original estaban formadas por dos pantallas macizas de hormigón armado conformando, junto con el forjado inferior, una célula triangular. Sobre las mismas se ha realizado un macizado de hormigón, para acoger los anclajes de una de las familias de pretensado exterior dispuesto en el interior del cajón, y los quiebros en planta y en alzado de ambas familias, a su paso por las pilas. Adicionalmente, se ha reforzado la armadura de las pantallas originales por un doble motivo: por un lado, por el incremento de esfuerzos de desequilibrio en pila, al aumentar el ancho de la calzada y por tanto el peso propio y la sobrecarga (sobre todo en las pilas extremas 1 y 5 , donde el desequilibrio es mayor), y por otro, para suplementar armadura cortada, al realizar los taladros necesarios para el paso de las familias de pretensado exterior.

Para el cálculo de la armadura a suplementar, se ha supuesto un doble mecanismo resistente en diafragma de pila: por un lado, un sistema biela-tirante en la célula triangular que recoge el momento de desequilibrio, y por otro un mecanismo de torsión, que provoca la aparición de cortantes en las almas laterales y central (figura 13). A partir del modelo de elementos finitos, 

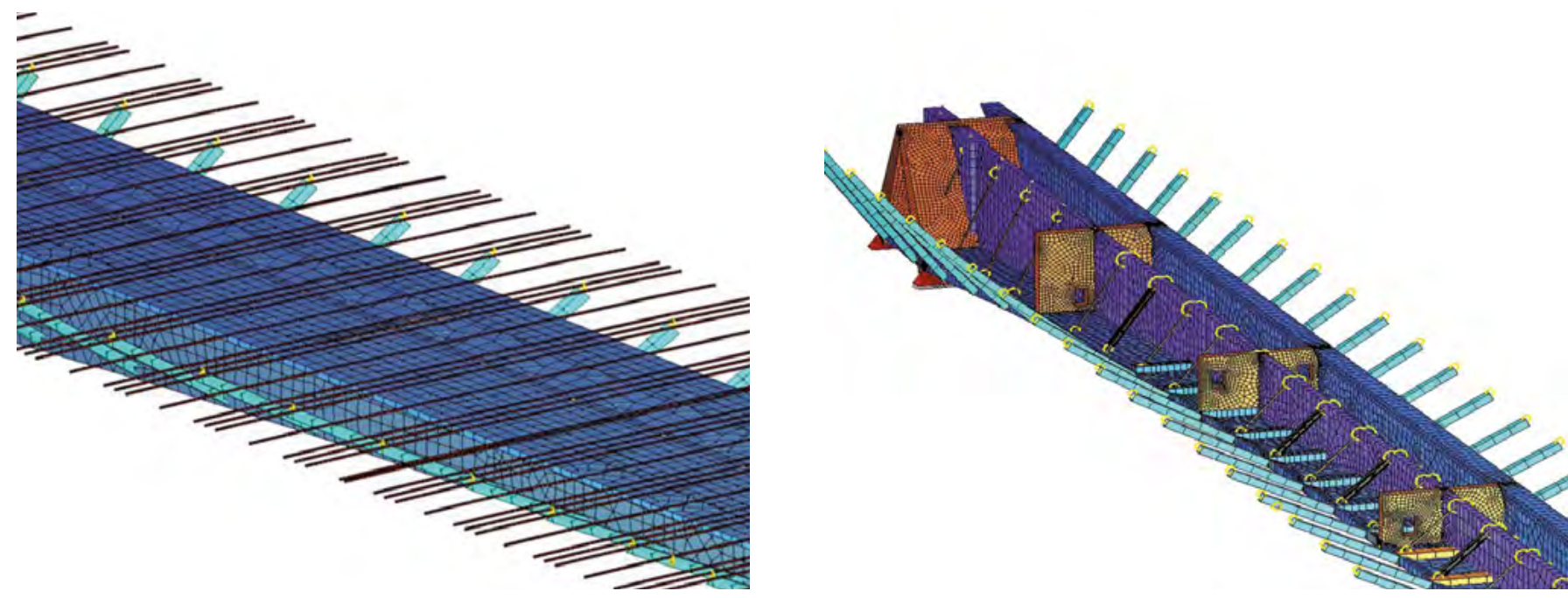

Figura 12. Viaducto de San Pedro de la Ribera. Modelo para el cálculo transversal.

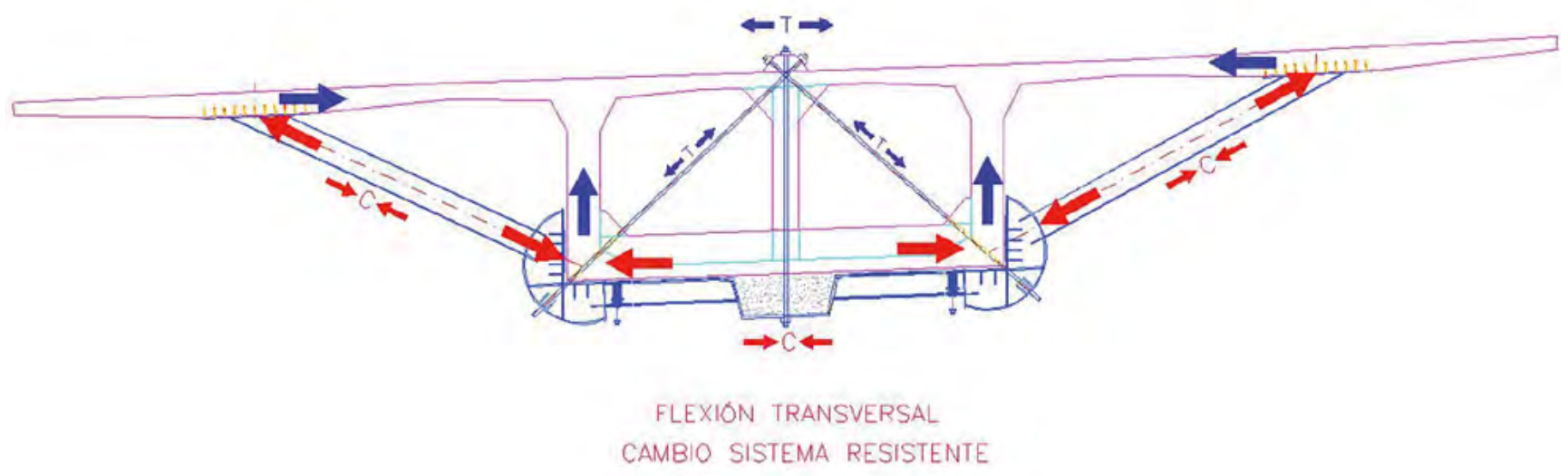

Figura 13. Viaducto de San Pedro de la Ribera. Mecanismo de flexión transversal.

por integración de tensiones resultantes para una carga uniforme, se dedujo que un $55 \%$, aproximadamente, de la carga se resistía por el primer mecanismo, y un $45 \%$ por el segundo, que es un reparto usual para este tipo de elementos estructurales [7],[8].

\subsection{Puente de Rande 6}

El puente de Rande se construyó a finales de 1970. Es un puente atirantado de $400 \mathrm{~m}$ de vano central y $23.46 \mathrm{~m}$ de anchura. En 2015 se ha procedido a la ampliación de su anchura suplementando la estructura existente con sendas nuevas estructuras en los laterales del tablero. Estos nuevos "tableros" se soportan con nuevos tirantes que se anclan en la coronación de las torres existentes. Para ello se dispone una nueva pieza metálica sobre la torre existente a la que se anclan los nuevos tirantes.

Uno de los principales criterios de proyecto de la ampliación fue mantener el mismo comportamiento estructural en el tablero existente una vez que la ampliación se hubiera realiza-

6 Proyecto redactado por MC-2 y Manuel Juliá. do; otro, fue el de que no se incrementaran los esfuerzos en los tirantes y en el tablero existente o, en su defecto, que dicho incremento fuera liviano y asumible, por supuesto. Para alcanzar ambas cosas había que conseguir minimizar la transferencia de cargas, fundamentalmente de flexíón y compresión, de la ampliación a la estructura existente.

Este es uno de los motivos "estructurales" por el que se decidió que la plataforma de la ampliación fuera soportada por sendas estructuras laterales, una a cada lado del tablero existente, a las que se conectan nuevos tirantes que se anclaban en estos nuevos tableros. Para conseguir el objetivo de reducir la transferencia de carga al tablero existente se definió una conexión entre ambas estructuras mediante una rótula esférica que permitía el giro relativo entre ellas, pero transmitía axiles y cortantes.

El esquema estructural que resulta es peculiar y, a la vez, beneficioso para el tablero existente puesto que las cargas permanentes y las sobrecargas de uso del nuevo tablero lo descargan. En efecto, la acción de estas cargas en los nuevos tableros es excéntrica (figura 14) y genera una fuerza ascendente sobre el tablero existente que reduce su carga vertical y transfiere parte de la carga de los tirantes existentes a los nuevos. Aun 


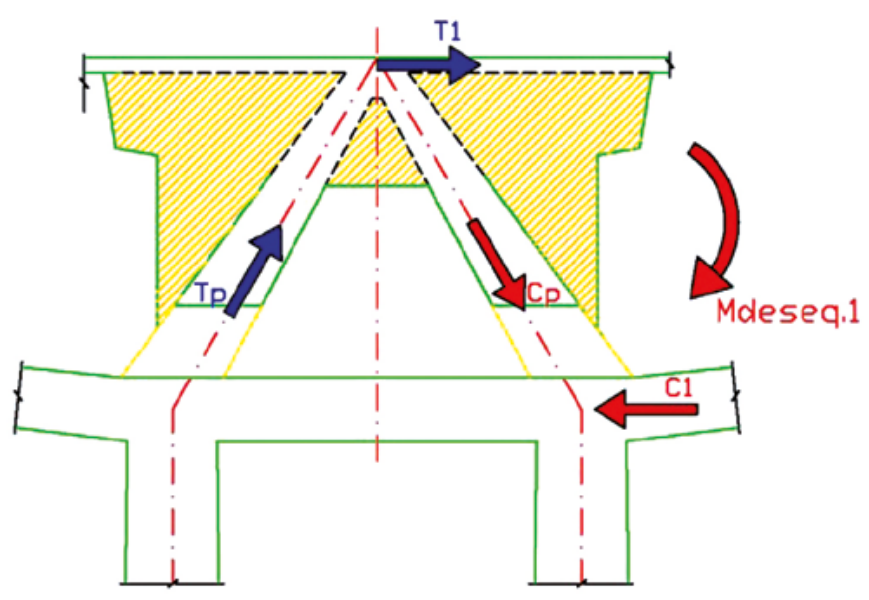

MECANISMO 1

BIELA - TIRANTE

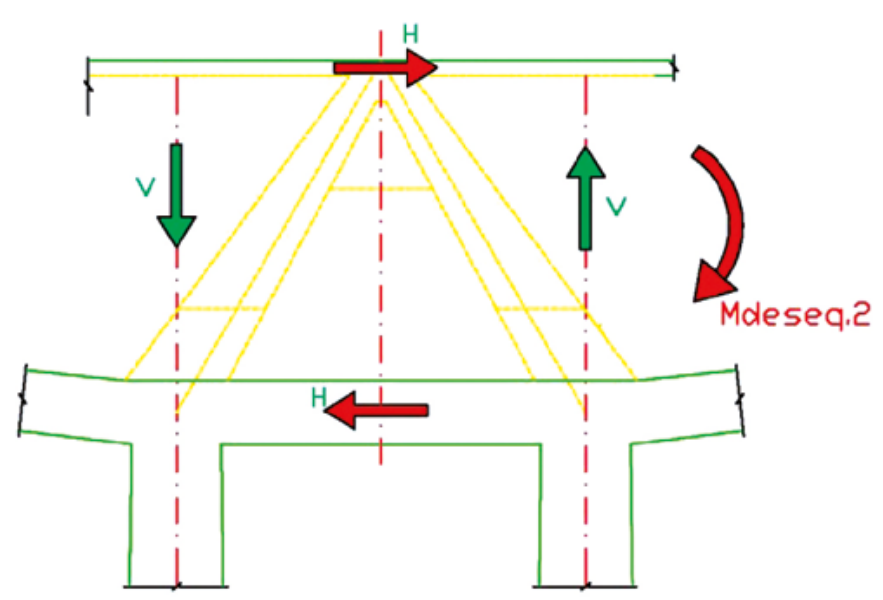

MECANISMO 2

CORTANTE EN ALMAS

Figura 14. Viaducto de San Pedro de la Ribera. Conexión tablero-fuste de pila.

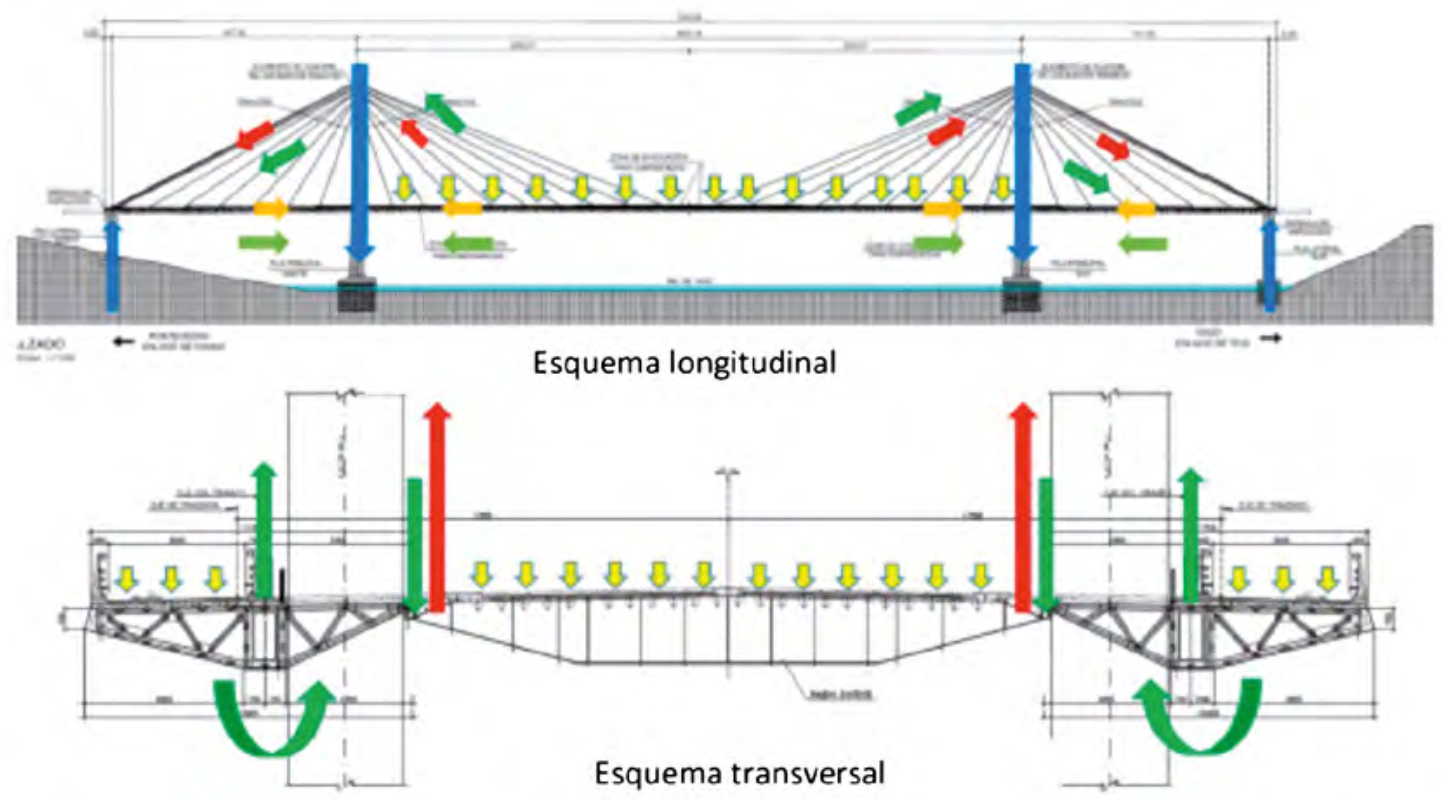

Figura 15. Puente de Rande. Esquema estructural.

así, se tuvo que proceder al refuerzo de algunas secciones del tablero que resultaban localmente algo más solicitadas.

Para afectar lo menos posible a las torres existentes se proyectó una estructura metálica que recibía los nuevos tirantes (figura 15). Así se conseguía no tener que ejecutar los nuevos anclajes de los nuevos tirantes en el antiguo pilono. La nueva estructura de acero se coloca sobre la torre existente a la que transmite sus cargas verticales mediante unos apoyos esféricos. Las cargas horizontales que provienen de las fuerzas longitudinales no equilibradas y de las transversales debidas al viento, por ejemplo, se transmiten mediante unos apoyos horizontales situados unos $11 \mathrm{~m}$ por debajo de los apoyos verticales referidos.

Para la validación de los elementos estructurales se empleó un modelo evolutivo que contemplaba 269 fases de construcción, incluidas las de ejecución del puente original, para así poder evaluar con suficiente aproximación las cargas en los tiran- tes y poder definir correctamente los tesados a dar en cada fase.

Para verificar la validez del tablero existente se consideró la contribución elastoplástica de los materiales y se realizaron cálculos no lineales considerando no linealidades geométricas y mecánicas.

Por último, hay que resaltar que, para la valoración del efecto del viento sobre la estructura se ha empleado un túnel de viento, sobre todo para conocer la potencial incidencia de algún fenómeno aeroelástico.

\subsection{Puente del Centenario ${ }^{7}$}

El puente del Centenario en Sevilla es un puente atirantado construido en 1991, de 256 m de luz y 22 m de ancho de pla-

7 Proyecto elaborado por FHECOR-IDEAM. 


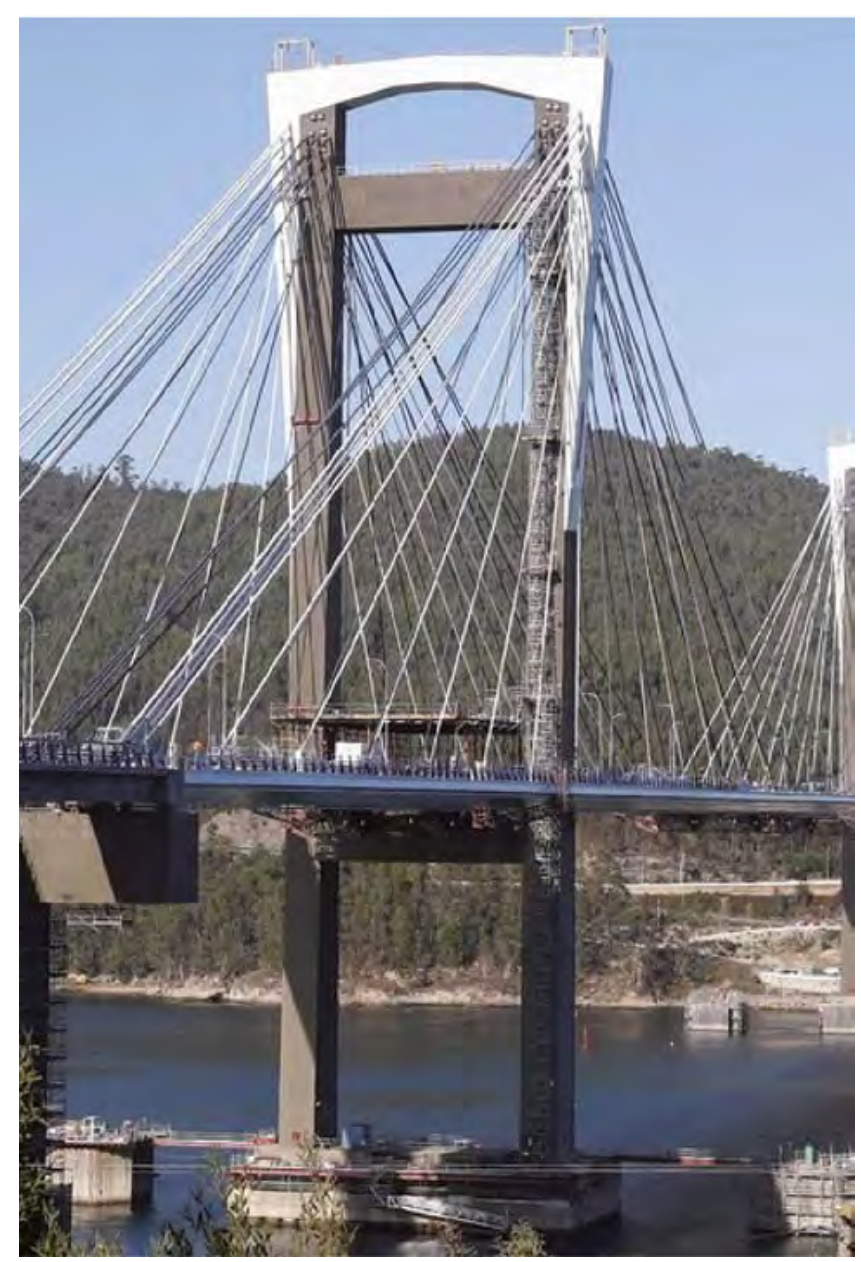

Figura 16. Puente de Rande. Recrecido de la torre.

taforma, cuyo tablero es de hormigón prefabricado. En 2021 se acometerá la Sustitución de los tirantes. Está previsto que, además de cambiar los tirantes, se aproveche para realizar la ampliación de la plataforma desde los 5 carriles existentes a 6 , en primera fase, y a 10 , en una segunda y definitiva fase. La actuación propuesta consiste en retirar los tirantes existentes, previa colocación de unos nuevos que transmiten la carga a unos nuevos fustes que se adosan y trabajan conjuntamente a los pilares que conforman las torres originales del puente. También se propone la mejora del terreno de cimentación para que soporte adecuadamente las cargas de los nuevos elementos adicionales que se propone construir.

El principal criterio que guía el proyecto es el de no sobrepasar en ningún elemento existente del puente el estado tensional generado en la configuración actual. Esto es así por dos motivos: porque la actuación "madre" de toda la obra es la retirada de los tirantes existentes que ya son obsoletos; y porque el tablero existente es de dovelas prefabricadas con juntas secas y, por tanto, no puede admitir la potencial aparición de tracciones, aunque fueran mínimas.

Para tratar de evitar la aparición de sobrecargas en los elementos portantes existentes se eligió la secuencia de construcción que se presenta de forma resumida e indicativa a continuación:

- Mejora del terreno bajo las cimentaciones de los pilonos existentes.
- Construcción de unos nuevos fustes adosados a los existentes en los que se anclarán los nuevos tirantes al llegar a la torre.

- Retirada de barreras, aceras y pavimentos y así descargar el tablero existente.

- Colocación y tesado de unos tirantes provisionales que se anclan en los nuevos fustes y en el tablero existente.

- Colocación de las nuevas piezas metálicas del tablero y colocación y tesado de los nuevos tirantes anclados en el nuevo tablero y en los nuevos fustes de las torres.

- Retesado de tirantes nuevos y destesado y retirada de los antiguos.

- Colocación de la carga muerta y retesado final de los nuevos tirantes.

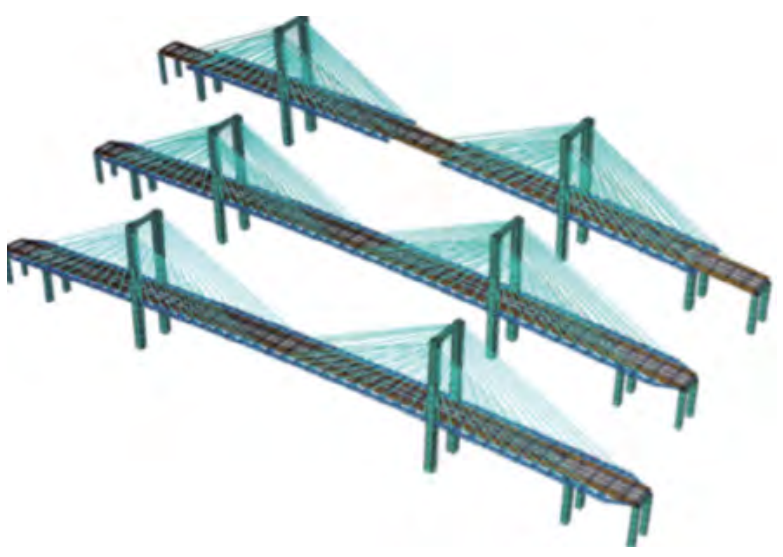

Figura 17. Puente del Centenario. Modelos estructurales.

Las 141 fases de construcción en que se representó la secuencia constructiva seleccionada fueron modelizadas con modelos de modelos de barras y cables que representan los elementos estructurales del puente: dovelas pretensadas, pilonos, riostras, costillas y tirantes (figuras 16 y 17). Las vigas pretesas longitudinales que apoyan en las riostras transversales no se consideran más que a efectos de carga muerta, por no contribuir al comportamiento global del tablero. Se ha modelizado la geometría real de los elementos existentes, así como la de los nuevos elementos. Para la definición de la estructura existente se ha tomado la información contenida en los planos del proyecto modificado y en el resto de los documentos de referencia del proyecto.

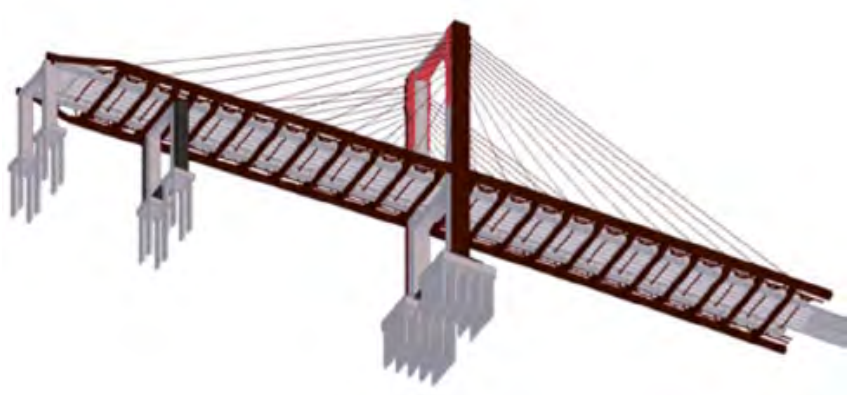

Figura 18. Puente del Centenario. Modelo BIM.

Como se ha comentado el proceso constructivo seleccionado, y las secuencias y valores de los tesados de los nuevos 


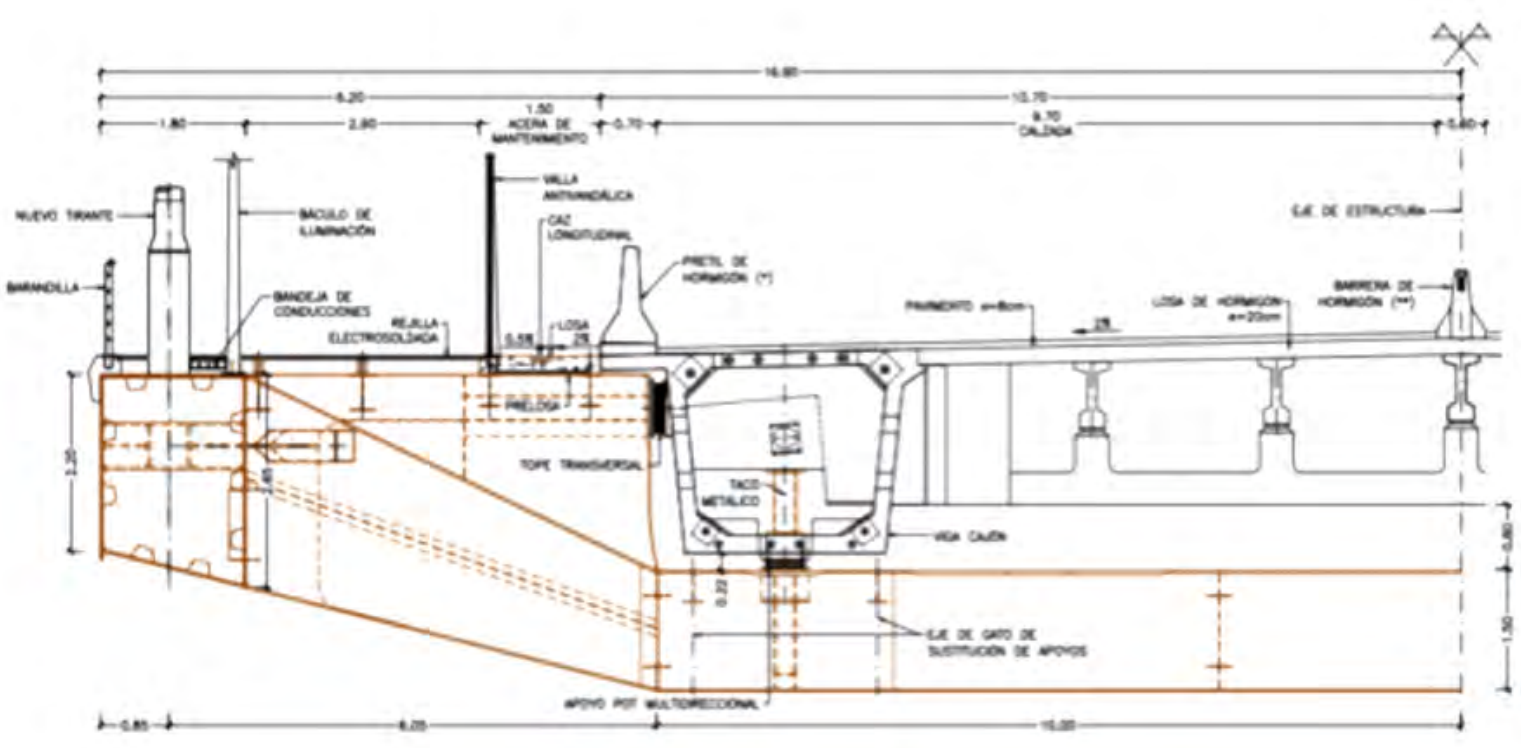

Figura 19. Puente del Centenario. Sección transversal.

tirantes pretendian que no se produjera ningún incremento de esfuerzos relevante en la estructura prefabricada del tablero existente (figura 18). Sin embargo, al haber conexión horizontal entre los nuevos elementos mixtos de la ampliación y el hormigón del tablero existente, el tablero existente recogía - por compatibilidad de movimientos - parte del axil de compresión y de la flexión inducida que se provocaba en el nuevo tablero al colocar las nuevas piezas del tablero y tesar los nuevos cables. Por eso se decidió disponer juntas cada 30-40m en los nuevos cordones metálicos; así se "aclaraba" el camino de las cargas y el esquema estructural resultante: el tablero de hormigón existente se apoya en las vigas transversales metálicas que transmiten la carga vertical a los nudos de la viga lateral metálica que recibe el tiro inclinado de los tirantes; las vigas longitudinales metálicas, que ven cortada su continuidad longitudinal cada $30-40 \mathrm{~m}$, se conectan horizontalmente a las vigas artesa de hormigón existente y les transmiten la compresión producida por la inclinación de los tirantes.

Para el cálculo de las torres se empleó un modelo 3D de doble barra: unas representan los fustes existentes y la paralela simula el nuevo fuste. Se dispusieron conexiones rígidas a tres alturas de los pilonos (zona superior, zona del tablero y otra intermedia). Con este modelo se analizó el reparto de esfuerzos entre ambos fustes y se evaluó el rasante que sirvió para dimensionar las uniones entre ellos, que se diseñaron pretensadas.

Por último, hay que resaltar que, como en otros puentes, se hizo un ensayo en túnel de viento para ajustar los valores de los coeficientes de arrastre a emplear en la caracterización de la acción del viento. También se evaluó con el ensayo la aptitud aeroelástica del puente, resultando satisfactorias las comprobaciones realizadas.

\section{Agradecimientos}

Los autores desean agradecer la contribución para la elaboración de este artículo a los autores de los proyectos de los puentes mencionados: Hugo Corres (FHECOR, puente de los Santos); Álvaro Serrano (MC2, puente de Rande); Luis Matute (IDEAM, puente del Centenario) y José Romo (FHECOR, puente del Centenario);y, todo el equipo técnico de TORROJA INGENIERIA, autores del proyecto de ampliación del viaducto de San Pedro.

\section{Referencias}

[1] Buckland, P.G., Matson, D. (2006) Increasing the load capacity of major bridges, Proceedings of the IABSE Symposyum Zurich, IABSE, pp99-106.

[2] Buckland, P.G. (2003) Increasing the load capacity of suspension bridges, Journal of Bridge Engineering, ASCE, Vol. 8, No. 5, pp 288-296.

[3] Torroja, J.A., Simón-Talero, J.M., Hernández, A., Navarro, A. (2011) Widening of the San Pedro de la Ribera Bridge, Hormigón y Acero, 62(260):11-45.

[4] Torroja, J.A., Simón-Talero, J.M., Hernández, A. (2011) Doubling the width of the platform of the San Pedro de la Ribera Bridge, PCI Journal, PCI, Vol Summer 2011, 2011, pp 1-16.

[5] Simón-Talero, J.M. (2009) Ampliación del puente de San Pedro de la Ribera. Jornada Técnica sobre diseño y concepción de puentes de carretera. Estética, eficiencia e innovación. ATC - Asociación Técnica de la Carretera. Madrid.

[6] Corres, H., García-Arango, I.,Pérez, A., Ramos, O., Domínguez, O., Peset, L. (2014) Ampliación del puente de Los Santos en la A-8 de 12.0 a 24.6 m sin cortes de tráfico, Hormigón y Acero, 65(273);199-220.

[7] Martínez-Calzón, J., Julia, M., Serrano, A., Gómez, M. (2008) Widening of the cable.stayed bridge over the Rande Strait, Spain, Structural Engineering International, IABSE, Vol 4, 2008, pp 314-317.

[8] Serrano, A., Rupérez, M., Alonso, J.C., Rodríguez, D. (2019) Widening of the cable.stayed bridge over the Rande Strait, Spain, Structural Engineering International, IABSE.

[9] Bernardo, H., Tarquis, F.,Lucas, C., Viartola, L.M. (2019) Rande Bridge widening: a $400 \mathrm{~m}$ span cable stayed bridge expansion, Practice Periodical Structurral Design and Construction, ASCE. 